\title{
An approximation to the Woods-Saxon potential based on a contact interaction
}

\author{
C. Romaniega ${ }^{1}$, M. Gadella ${ }^{1}$, R.M. Id Betan ${ }^{2,3,4}$ and L.M. Nieto ${ }^{1}$ \\ 1 Departamento de Física Teórica, Atómica y Óptica and IMUVA, Universidad de Valladolid, 47011. Valladolid, Spain. \\ 2 Instituto de Física Rosario (CONICET-UNR), Bv. 27 de Febrero 210 bis, S2000EZP Rosario. Argentina. \\ 3 Facultad de Ciencias Exactas, Ingeniería y Agrimensura (UNR), Av. Pellegrini 250, S2000BTP Rosario. Argentina. \\ ${ }^{4}$ Instituto de Estudios Nucleares y Radiaciones Ionizantes (UNR), Riobamba y Berutti, S2000EKA Rosario. Argentina
}

Received: date / Revised version: date

\begin{abstract}
We study a non-relativistic particle subject to a three-dimensional spherical potential consisting of a finite well and a radial $\delta-\delta^{\prime}$ contact interaction at the well edge. This contact potential is defined by appropriate matching conditions for the radial functions, thereby fixing a self adjoint extension of the non-singular Hamiltonian. Since this model admits exact solutions for the wave function, we are able to characterize and calculate the number of bound states. We also extend some well-known properties of certain spherically symmetric potentials and describe the resonances, defined as unstable quantum states. Based on the Woods-Saxon potential, this configuration is implemented as a first approximation for a mean-field nuclear model. The results derived are tested with experimental and numerical data in the double magic nuclei ${ }^{132} \mathrm{Sn}$ and ${ }^{208} \mathrm{~Pb}$ with an extra neutron.
\end{abstract}

PACS. 02.30.Em Potential theory - 03.65.-w Quantum mechanics

\section{Introduction}

In one-dimensional non-relativistic quantum mechanics, point potentials or potentials supported on one or a discrete collection of points, like the Dirac delta interactions, have deserved considerable attention recently (see [1] and references quoted therein). These potentials are often exactly solvable and therefore, provide a good insight for some quantum phenomena like scattering. In addition, they serve as a fair approximation for various types of interactions, as very short range interactions between a single particle and a fixed heavy source as well as a contact interaction in the centre of mass of two particles. This is the origin of the name contact potentials. They also function as suitable approximations when the particle wavelength is much larger than the range of the potential. In spite of their simplicity, they have a vast amount of applications in modelling real physical systems, as we can see for instance in a recent review [1], some books [2, 3 and the references therein. The landmark example in solid state physics is a limiting case of the Kronig-Penney model in which a countably infinite set of Dirac delta interactions are periodically distributed along a straight line [4, 5, 6]. Other examples of physical interest are the following: a Bose-Einstein condensation in a harmonic trap with a tight and deep "dimple" potential, modelled by a Dirac delta function [7] a non-perturbative study of the entanglement of two directed polymers subject to repulsive interactions given by a Dirac delta potential [8]; light propagation in a one-dimensional realistic dielectric superlattice, modelled by means of a periodic array of these functions for the cases of transverse electric, transverse magnetic, and omnidirectional polarization modes 9 .

From a purely mathematical point of view, one-dimensional contact potentials have been studied as self adjoint extensions of the kinetic energy operator $-d^{2} / d x^{2}[3,10$. This approach has been used to construct several onedimensional models which go beyond the Dirac delta potential [11. A discussion on the physical meaning of the one-dimensional contact potentials constructed as self adjoint extensions of the kinetic energy operator is given in [12] It is remarkable that, inspired in the physics of contact interactions, new mathematics has been developed [13.

In the present manuscript we consider a three-dimensional spherically symmetric potential. Although the problem reduces to a one-dimensional one once the centrifugal term is included, the model can be more suitable for the implementation into realistic situations than the abovementioned one-dimensional results. As will be explained later, this potential would serve as an approximation for a nuclear model.

Focusing on the contact interaction, and for reasons to be exposed below, in this paper we are going to analyse a linear combination of two independent interactions: a Dirac delta and a $\delta^{\prime}$ potential, both supported on a hollow sphere of radius $x_{0}$. Thus, this interaction is represented by a potential of the form 


$$
V(x)=v_{1} \delta\left(x-x_{0}\right)+v_{2} \delta^{\prime}\left(x-x_{0}\right), \quad x \geq 0, x_{0}>0 .
$$

As mentioned earlier, spherically symmetric three-dimensional Schrödinger equations admit a one-dimensional counterpart if the orbital angular momentum is left fixed, which is the so called radial equation. When working with the radial equation, the $\delta-\delta^{\prime}$ interaction supported on the sphere become a one-dimensional $\delta-\delta^{\prime}$ interaction supported on the point $x_{0}>0$. A self adjoint determination for the Hamiltonian $H=-d^{2} / d x^{2}+V(x)$ with the potential (1) in one dimension has been discussed in [14]. Although the definition for the $\delta$ interaction is universally accepted, this is not the case for the $\delta^{\prime}$ term, for which at least two definitions are consistent with its desirable properties [15, 16]. The determination and sense of the $\delta^{\prime}$ interaction is given by the use of proper matching conditions for the wave functions at $x_{0}$. These matching conditions determine a domain in which the Hamiltonian with the $\delta^{\prime}$ interaction is self-adjoint [10]. In this paper, following the lines developed in [14,17, we shall use the so called local $\delta^{\prime}$ interaction since it is compatible with the $\delta$ potential in such a way that the total Hamiltonian $H=-d^{2} / d x^{2}+V(x)$ is self adjoint. As we shall see, since both interactions are supported on the same point, the proposed model naturally leads to this determination which is beautifully given by a proper choice of matching conditions for the wave functions at $x_{0}$.

Accordingly, the same choice of the Hamiltonian $H=$ $-d^{2} / d x^{2}+V(x)$ has been used in a study in which the interaction (1) plays a fundamental role: the approximation of a system formed by two thin plates in order to describe the quantum vacuum fluctuations in the presence of boundaries: the Casimir effect [18. In this context, the addition of the $\delta^{\prime}$ term can be useful when dealing with boundary conditions (BC). Robin BC can be obtained as a finite limit of the previous interaction. Although Dirichlet $\mathrm{BC}$ can also be reached with the $\delta$ potential alone, the strength going to infinity could be troublesome for the Casimir self-energy of a sphere [19,20]. Some other discussions on properties of contact potentials are given in [15, [21,22, 23] and their use in supersymmetric quantum mechanics is shown in [24,25, 26, 27,28 and references quoted therein. We do not intend to be exhaustive and just mention recent literature.

As pointed out above, we also investigate its possible use as a mean field nuclear model, which has been a motivation to study this particular case. Within the Woods-Saxon approximation, the Dirac delta interaction has been used for the calculation of resonant parameters and energy spectrum in 29. Recently, it has also been employed to study the spectral function of the unbound nucleus ${ }^{25} \mathrm{O}$ 30. In the latter case, a comparison of the spectrum obtained between the $\delta$ potential versus the nuclear non-singular mean-field is performed. In this paper we also test the results obtained with the regular meanfield potential in two nuclei, ${ }^{133} \mathrm{Sn}$ and ${ }^{209} \mathrm{~Pb}$. The main advantage of this approach is that the wave function can be easily solved in terms of well-known special functions.
This enables us to derive analytic properties of the neutron energy levels structure.

The article is organized as follows. In Section 2, we introduce the potential under consideration, which is written in a language that will fit well for the future application to a nuclear model. The radial $\delta$ and $\delta^{\prime}$ terms appear here explicitly. In Section 3, we derive the secular equation for which the solutions give the bound states. In Section 4, we study the existence and localization of bound states, giving some rigorous results. We study the resonances arisen in our example in Section 5 . The analysis of resonances is necessary because most of the known quantum states in nuclear or atomic physics are unstable. These findings are tested with the nuclei ${ }^{133} \mathrm{Sn}$ and ${ }^{209} \mathrm{~Pb}$ in section 6, the latter being of some relevance in particle astrophysics 31. Concluding remarks, and two appendices, one devoted to some comments on the self adjointness of the Hamiltonian and the other to the proofs of the main results of the text, give an end to this paper.

\section{Model and motivation}

Along this section, we develop the quantum model under study. We have preferred to use a language that makes it suitable for possible applications, particularly in nuclear physics. Nevertheless, a non-relativistic quantum particle in a spherical well with a contact interaction in the edge can be analysed within this framework. In order to make the link between the strength which appears in the radial $\delta-\delta^{\prime}$ interaction and real physical parameters 32,33 , 34, we start with the following three-dimensional single particle Hamiltonian in the centre of mass system,

$$
H(\mathbf{r})=-\frac{\hbar^{2}}{2 \mu} \nabla_{\mathbf{r}}^{2}+U_{0}(r)+U_{\mathrm{so}}(r)(\mathbf{L} \cdot \mathbf{S})+U_{\mathrm{q}}(r) .
$$

The reduced mass is denoted by $\mu$, being $U_{0}(r), U_{\text {so }}(r)$, and $U_{\mathrm{q}}(r)$, essentially, the Woods-Saxon potential [35], its first and second derivative, respectively,

$$
\begin{aligned}
& U_{0}(r)=-V_{0} f(r)=-V_{0} \frac{1}{1+e^{(r-R) / a}}, \\
& U_{\mathrm{so}}(r)=\frac{V_{\mathrm{so}}}{\hbar^{2}} f^{\prime}(r)=-\frac{V_{\mathrm{so}}}{a \hbar^{2}} \frac{e^{(r-R) / a}}{\left(1+e^{(r-R) / a}\right)^{2}}, \\
& U_{\mathrm{q}}(r)=V_{\mathrm{q}} f^{\prime \prime}(r)=-\frac{V_{\mathrm{q}}}{a^{2}} \frac{e^{(r-R) / a}\left(1-e^{(r-R) / a}\right)}{\left(1+e^{(r-R) / a}\right)^{3}} .
\end{aligned}
$$

Since we only study configurations with an extra neutron, the Coulombic potential is not included in the Hamiltonian. The strengths $V_{0}$ and $V_{\text {so }}$ of the Woods-Saxon potential and the spin-orbit, respectively, are positive defined in order to reproduce the experimental magic number [36, while the sign of $V_{\mathrm{q}}$ can be selected to fit with the experimental data. This second derivative may be employed as a form factor in the transition operator for the quadrupolar electric transition E2 in the Interacting Boson Approximation model 37 . The nuclear radius $R$ is parametrized 
in terms of the nuclear mass $A=N+Z$ as $R=r_{0} A^{1 / 3}$, being $r_{0}$ constant and $N, Z$ the number of neutrons and protons, respectively. The parameter $a$ gives the thickness of the surface of $f(r)$. The nuclear shell model considers $N$ or $Z$ as a magic number, and optimized all these parameters in a way to reproduce, as well as possible, the low-lying energy levels of the nuclei with one extra neutron or proton 38. Typical values for the parameters are $r_{0}=1.27 \mathrm{fm}, a=0.7 \mathrm{fm}, V_{0}=51 \pm 33(N-Z) / A \mathrm{MeV}$, with $+(-)$ for proton (neutron) 39 .

Going back to the Hamiltonian (2), we rewrite the kinetic operator in terms of the orbital angular momentum $\mathbf{L}$ and the radial coordinates as

$$
-\frac{\hbar^{2}}{2 \mu} \nabla_{\mathbf{r}}^{2}=-\frac{\hbar^{2}}{2 \mu}\left[\frac{1}{r^{2}} \frac{\partial}{\partial r}\left(r^{2} \frac{\partial}{\partial r}\right)-\frac{\mathbf{L}^{2} / \hbar^{2}}{r^{2}}\right] .
$$

The eigenfunctions of the corresponding three-dimensional stationary Schrödinger equation are factored into a radial $u_{n \ell j}(r) / r$ and angular part $\mathcal{Y}_{\ell j m}(\theta, \phi)$. The latter fulfils

$$
\mathbf{L}^{2} \mathcal{Y}_{\ell j m}(\theta, \phi)=\hbar^{2} \ell(\ell+1) \mathcal{Y}_{\ell j m}(\theta, \phi), \quad(\mathbf{L} \cdot \mathbf{S}) \mathcal{Y}_{\ell j m}(\theta, \phi)
$$

The function $\mathcal{Y}_{\ell j m}(\theta, \phi)$, a linear combination of spherical harmonics $Y_{\ell m}(\theta, \phi)$, is a simultaneous eigenfunction of the operators $\mathbf{L}^{2}, \mathbf{S}^{2}, \mathbf{J}^{2}=(\mathbf{L}+\mathbf{S})^{2}$ and $J_{z}[39$. We have also defined $\xi_{\ell j}=\frac{1}{2}\left(j(j+1)-\ell(\ell+1)-\frac{3}{4}\right)$, that is

$$
\xi_{\ell j}=\left\{\begin{array}{c}
\frac{\ell}{2} \quad \text { for } j=\ell+\frac{1}{2} \\
-\frac{(\ell+1)}{2} \text { for } j=\ell-\frac{1}{2}
\end{array}\right.
$$

where $\ell$ takes values in the non-negative integers $\mathbb{N}_{0}$. For $\ell=0$ the only possibility is $j=1 / 2$ so $\xi_{0 j}=0$. Using the above relations, the radial part fulfils $H u_{n \ell_{j}}(r)=$ $E_{n \ell_{j}} u_{n \ell_{j}}(r)$ where

$H=\frac{-\hbar^{2}}{2 \mu}\left[\frac{d^{2}}{d r^{2}}-\frac{\ell(\ell+1)}{r^{2}}\right]-V_{0} f(r)+V_{\mathrm{so}} \xi_{\ell j} f^{\prime}(r)+V_{\mathrm{q}} f^{\prime \prime}(r)$.

Observe that the spin-orbit interaction is defined without the $1 / r$ factor as usual [40,41. This may be done since the nuclear spin-orbit does not have the same origin as the one in the atom, and yet it is not well understood. In table 1 we show that the change has not effect on the $s$ partial wave, as it should be, while it is more pronounced as the principal quantum number increases for $\ell=1$. Since the difference can be absorbed in the effective strength $V_{\text {so }}$, the $1 / r$ term is omitted in our definition of the spin-orbit form factor.

Finally, in order to reach the explicit form of the effective potential used in section 6 , we take the limit $a \rightarrow 0^{+}$. Note that

$$
\lim _{a \rightarrow 0^{+}} U_{0}(r)=\left\{\begin{array}{cc}
-V_{0} & \text { if } r<R \\
-V_{0} / 2 & \text { if } r=R \\
0 & \text { if } r>R
\end{array}\right\}=V_{0}[\theta(r-R)-1]
$$

Table 1. Neutron energy levels $(\mathrm{MeV})$ in the core of ${ }^{208} \mathrm{~Pb}$ using $1 / r V_{\text {so }} \xi_{\ell j} f^{\prime}(r)$ (second column) versus $V_{\text {so }} \xi_{\ell j} f^{\prime}(r)$ (third column) as the radial form factor of the spin-orbit interaction, with $a=0.7 \mathrm{fm}, r_{0}=1.27 \mathrm{fm}, V_{0}=44.4 \mathrm{MeV}$, and $V_{\mathrm{so}}=16.5 \mathrm{MeVfm}$.

\begin{tabular}{lll}
\hline State $\left(n \ell_{j}\right)$ & $E_{n \ell_{j}}^{*}$ & \multicolumn{1}{l}{$E_{n \ell_{j}}$} \\
\hline $0 s_{1 / 2}$ & -40.231 & -40.231 \\
$0 p_{3 / 2}$ & -36.328 & -37.078 \\
$0 p_{1 / 2}$ & -35.928 & -34.901 \\
$1 s_{1 / 2}$ & -29.622 & -29.622 \\
$1 p_{3 / 2}$ & -23.471 & -25.029 \\
$1 p_{1 / 2}$ & -22.695 & -20.134 \\
$2 s_{1 / 2}$ & -15.299 & -15.299 \\
$2 p_{3 / 2}$ & -8.355 & -10.303 \\
$2 p_{1 / 2}$ & -7.413 & -3.370 \\
\hline
\end{tabular}

where $\theta(x)$ is the Heaviside step function. The function $f(r)$ can be seen as a distribution on a certain space of test functions, such as the Schwartz space. Then, if $\psi(r)$

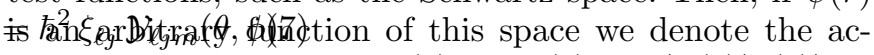
tion of the distribution $f(r)$ on $\psi(r)$ by $\langle\psi(r) \mid f(r)\rangle=$ $\int_{0}^{\infty} \psi^{*}(r) f(r) d r$. For the first derivative we obtain

$\lim _{a \rightarrow 0^{+}}\left\langle\psi(r) \mid \frac{d}{d r} f(r)\right\rangle=-\lim _{a \rightarrow 0^{+}}\left\langle\psi^{\prime}(r) \mid f(r)\right\rangle=-\left\langle\psi^{\prime}(r) \mid 1-\theta(r-R)\right\rangle$

This holds since the Dirac delta is the derivative of the Heaviside step function, from the point of view of distributions. Consequently,

$$
\lim _{a \rightarrow 0^{+}} V_{\mathrm{so}} \xi_{\ell j} f^{\prime}(r)=-V_{\mathrm{so}} \xi_{\ell j} \delta(r-R) .
$$

In the same way, we obtain the following expression for the second derivative:

$$
\lim _{a \rightarrow 0^{+}} U_{\mathrm{q}}(r)=\lim _{a \rightarrow 0^{+}} V_{\mathrm{q}} f^{\prime \prime}(r)=-V_{\mathrm{q}} \delta^{\prime}(r-R) .
$$

In view of these considerations, the Hamiltonian (9) turns into

$H_{\text {sing }}=-\frac{\hbar^{2}}{2 \mu}\left[\frac{d^{2}}{d r^{2}}-\frac{\ell(\ell+1)}{r^{2}}\right]+V_{0}[\theta(r-R)-1]-V_{\mathrm{so}} \xi_{\ell j} \delta(r-R$

where the singular (contact) terms are already included. A comment on the $\delta^{\prime}$ contribution in $(14)$ is in order here. As explained in appendix A the $\delta$ - $\delta^{\prime}$ perturbation is defined using the formalism of self adjoint extensions of symmetric (formally Hermitian) operators with equal deficiency indices. This gives two options for the $\delta^{\prime}$ term. The former is a $\delta^{\prime}$ which is often called the non-local $\delta^{\prime} 15$. However, this choice is incompatible with the Dirac- $\delta$ [16], so that we have to use the other choice, the local $\delta^{\prime}$ interaction, which is defined by matching conditions established at the point supporting the interaction. From a distributional point of view, this is a generalization of the usual definition of the derivative of the delta, which has to be adapted to test functions with a discontinuity at $x_{0}$ and a discontinuity of their derivative at the same point [10. In any case, the $\delta^{\prime}$ 
perturbation is properly defined via matching conditions at $x_{0}$, as we have already mentioned.

Thus, bearing in mind that $R \gg a$, we can consider the above simplified one-dimensional Hamiltonian (14) as a mean-field potential to describe neutron energy levels. One of the main advantages is that the eigenvalue equation $H_{\text {sing }} u_{\ell}(r)=E_{n \ell_{j}} u_{\ell}(r)$ can be solved exactly for the wave function ${ }^{5}$ in terms of Bessel functions. Consequently, the main findings of the text are based on the properties of these functions.

\section{Solutions of the singular Schrödinger equation}

In this section, we determine the eigenfunctions of the singular Hamiltonian (14),

$\left[-\frac{d^{2}}{d r^{2}}+\frac{\ell(\ell+1)}{r^{2}}-\frac{2 \mu E}{\hbar^{2}}+\frac{2 \mu V_{0}}{\hbar^{2}}(\theta(r-R)-1)+\alpha \delta(r-\right.$

where

$$
\alpha=-\frac{2 \mu}{\hbar^{2}} V_{\mathrm{so}} \xi_{\ell j}, \quad \beta=-\frac{2 \mu}{\hbar^{2}} V_{\mathrm{q}} .
$$

At this point, we should remark that we may look to the parameters $\alpha$ and $\beta$ as two independent coefficients, with no relation whatsoever with any future application to a nuclear model. In this sense, equation (15) can be considered for a quantum particle subject to a spherical well with a $\delta$ - $\delta^{\prime}$ interaction at the edge.

The radial Schrödinger equation is defined on the interval $0 \leq r<\infty$. Due to the presence of the contact potential, we divide this semi-axis into two regions: $0 \leq r<R$ and $R<r$. We shall obtain the wave function in each region and then apply suitable matching conditions at $r=R$, thus defining the singular part of the Hamiltonian.

\subsection{Interior wave equation}

In the study of the solutions in the first region $0 \leq r<R$, we consider energy values $E>-V_{0}$. Hence, if we perform the transformations

$$
x=\gamma r \quad y_{\ell}(x)=u_{\ell}(r), \gamma=\frac{\sqrt{2 \mu\left(V_{0}+E\right)}}{\hbar}, x \in[0, \gamma R),
$$

then, equation (15) becomes a Riccati-Bessel differential equation:

$$
\frac{d^{2} y_{\ell}(x)}{d x^{2}}-\frac{\ell(\ell+1)}{x^{2}} y_{\ell}(x)+y_{\ell}(x)=0, \quad \ell \in \mathbb{N}_{0} .
$$

For each particular value of the orbital angular momentum $\ell$, the general solution is given by

$$
y_{\ell}(x)=\sqrt{x}\left(A_{\ell} J_{\ell+\frac{1}{2}}(x)+B_{\ell} Y_{\ell+\frac{1}{2}}(x)\right),
$$

\footnotetext{
${ }^{5}$ For simplicity, and when no confusion arises, we will use abbreviated notation such as $u_{\ell} \equiv u_{n \ell_{j}}$.
}

where $J_{\ell+1 / 2}(x)$ and $Y_{\ell+1 / 2}(x)$ denote the Bessel functions of first and second kind, respectively, being $A_{\ell}$ and $B_{\ell}$ arbitrary constants. For small values of the positive variable $x$, the asymptotic forms of the aforementioned Bessel functions are given by

$J_{\ell+\frac{1}{2}}(x) \sim \frac{\left(\frac{x}{2}\right)^{\ell+\frac{1}{2}}}{\Gamma\left(\ell+\frac{3}{2}\right)}, \quad Y_{\ell+\frac{1}{2}}(x) \sim-\frac{\Gamma\left(\ell+\frac{1}{2}\right)}{\pi}\left(\frac{2}{x}\right)^{\ell+\frac{1}{2}}$.

Hence, if we are looking for square integrable solutions, we should impose $B_{\ell}=0$ for $\ell \neq 0$, since $\sqrt{x} Y_{\ell+1 / 2}(x)$ behaves near zero as $x^{-\ell}$. For $\ell=0$, the radial Hamiltonian is not self adjoint, although it admits a one parameter family of self adjoint extensions 43. To fix one of them, we need to set boundary conditions at the origin for the functions $y_{0}(x)$ in the domain of the radial Hamiltonian. The simplest possibility is $y_{0}(0)=0$, which forces the choice $B_{0}=0$. In consequence, $B_{\ell}=0 \forall \ell \in \mathbb{N}_{0}$. On the other $R$ hand $\delta^{\prime}$ it $r$ is obyious after $(19)$ that $\sqrt{x} J_{\ell+\frac{1}{2}}(x)$ is zero at the origin and therefore square integrable on the finite interval considered. Consequently, the admissible solutions are just

$$
u_{\ell}(r)=A_{\ell} \sqrt{\gamma r} J_{\ell+\frac{1}{2}}(\gamma r), \quad r \in[0, R), \quad \ell \in \mathbb{N}_{0} .
$$

\subsection{Exterior wave equation}

For values of $r$ such that $R<r$, we have to solve the Schrödinger equation (15) for $V_{0}=0$. As we are looking for bound states, we require $E<0$. Then, we first proceed with the following changes:

$$
z=\kappa r, y_{\ell}(z)=u_{\ell}(r), \kappa=\frac{\sqrt{2 \mu|E|}}{\hbar}, z \in(\kappa R, \infty),
$$

which transform (15) into the following differential equation:

$$
\frac{d^{2} y_{\ell}(z)}{d z^{2}}-\frac{\ell(\ell+1)}{z^{2}} y_{\ell}(z)-y_{\ell}(z)=0, \quad \ell \in \mathbb{N}_{0} .
$$

For any value of $\ell$, the general solution of $(22)$ is given by

$$
y_{\ell}(z)=\sqrt{z}\left(C_{\ell} I_{\ell+\frac{1}{2}}(z)+D_{\ell} K_{\ell+\frac{1}{2}}(z)\right) .
$$

Here, $I_{\ell+1 / 2}(z)$ and $K_{\ell+1 / 2}(z)$ are the modified Bessel functions of first and second kind, respectively, being $C_{\ell}$ and $D_{\ell}$ arbitrary constants. Again, if we are looking for square integrable solutions, we need to know the asymptotic behaviours of these functions for large values of $z$, which are,

$$
I_{\ell+\frac{1}{2}}(z) \sim \frac{e^{z}}{\sqrt{2 \pi z}}, \quad K_{\ell+\frac{1}{2}}(z) \sim \sqrt{\frac{\pi}{2 z}} e^{-z} .
$$

Accordingly, the solution (23) is square integrable if, and only if, $C_{\ell}=0$. In this way, the only possible contribution comes from the second term, so that

$$
u_{\ell}(r)=D_{\ell} \sqrt{\kappa r} K_{\ell+\frac{1}{2}}(\kappa r), \quad r \in(R, \infty) \quad \ell \in \mathbb{N}_{0} .
$$


Once we have obtained the interior and exterior solutions, we need to link both of them at the point $r=R$ in an appropriate way.

\subsection{Matching conditions}

As established by the standard bibliography on the subject [10], there are requirements for the reduced radial function at the point $r=R$ which fix a self adjoint determination of the operator

$$
-\frac{d^{2}}{d r^{2}}+\frac{\ell(\ell+1)}{r^{2}}+\frac{2 \mu V_{0}}{\hbar^{2}}[\theta(r-R)-1]
$$

thus defining the final Hamiltonian of equation (15). These requirements are given by matching conditions relating the function $u_{\ell}(r)$ and its first derivative at the limit values of $R$. They can be written in terms of a $S L(2, \mathbb{R})$ matrix as [11,44,45.

$$
\left(\begin{array}{c}
u_{\ell}\left(R^{+}\right) \\
u_{\ell}^{\prime}\left(R^{+}\right)
\end{array}\right)=\left(\begin{array}{cc}
\frac{2+\beta}{2-\beta} & 0 \\
\frac{4 \alpha}{4-\beta^{2}} & \frac{2-\beta}{2+\beta}
\end{array}\right)\left(\begin{array}{c}
u_{\ell}\left(R^{-}\right) \\
u_{\ell}^{\prime}\left(R^{-}\right)
\end{array}\right), \quad \text { where }
$$

The function $u_{\ell}(r)$ is given by 20 and 25 . As already mentioned, there is a rigorous discussion on the self adjointness of the resulting Hamiltonian in appendix A. The matrix relation (26), together with (20) and (25), yields the following secular equation:

$\frac{\chi J_{\ell+\frac{3}{2}}(\chi)}{J_{\ell+\frac{1}{2}}(\chi)}=\frac{(2+\beta)^{2}}{(2-\beta)^{2}} \frac{\sigma K_{\ell+\frac{3}{2}}(\sigma)}{K_{\ell+\frac{1}{2}}(\sigma)}-\frac{8 \beta(\ell+1)}{(2-\beta)^{2}}+\frac{w_{\ell j}}{(2-\beta)^{2}}$.

We will denote the left-hand side by $\varphi_{\ell}(\chi)$ and the righthand $\operatorname{sid}^{1}$ by $\phi_{\ell}(\sigma)$, so (27) is written as $\varphi_{\ell}(\chi)=\phi_{\ell}(\sigma)$. For simplicity, we have introduced the following auxiliary variables

$$
\chi=v_{0} \sqrt{1-\varepsilon}, \quad \sigma=v_{0} \sqrt{\varepsilon},
$$

and defined the dimensionless parameters, $v_{0}, w_{\ell j}$ and the relative energy $\varepsilon$ as

$v_{0}=\sqrt{\frac{2 \mu R^{2} V_{0}}{\hbar^{2}}}>0, \quad w_{\ell j}=\frac{-8 \mu V_{\mathrm{so}} \xi_{\ell j} R}{\hbar^{2}}, \quad \varepsilon=|E| /$

The secular equation (27) does not admit closed-form solutions for the energy of bound states and it will be analyzed in the forthcoming section.

\section{General properties of the bound state structure}

In the previous section we have established the matching conditions that radial wave functions must fulfil so that the $\delta$ and the local $\delta^{\prime}$ interactions are well defined. With

\footnotetext{
${ }^{1}$ Observe that $\phi_{\ell}(\sigma)$ also depends explicitly on $j$ and $\beta$.
}

this, in the present section we consider the whole Hamiltonian (15) in order to study the existence and properties of bound states. Moreover, in section 4.1 we consider the cases for which the matching conditions are ill defined and we give a simplified secular equation for large-parameter configurations in section 4.2 .

Before proceeding with our presentation, let us denote by $j_{\lambda, s}$ the $s$-th strictly positive zero of $J_{\lambda}(x), \lambda>0$. As is well known, these zeros satisfy

$$
j_{\lambda, 0} \equiv 0<j_{\lambda, s}<j_{\lambda+1, s}<j_{\lambda, s+1}, \quad s \in \mathbb{N} .
$$

We begin with a result concerning the existence and number of bound states, whose proof is given in appendix B.1.

Theorem 1 If for any value $\ell \in \mathbb{N}_{0}$ such that $\ell \leq \ell_{\max }$ the following inequality holds

$$
w_{\ell j}>-\left((\beta-2)^{2}+2 \ell\left(\beta^{2}+4\right)\right),
$$

there exists one, and only one, energy level with relative $u_{\ell}($ Rte $) g y-\lim _{x \rightarrow R^{ \pm}} u_{\ell}(x)$.

$$
\varepsilon_{s} \in\left(1-\frac{j_{\ell+1 / 2, s}^{2}}{v_{0}^{2}}, 1-\frac{j_{\ell+3 / 2, s-1}^{2}}{v_{0}^{2}}\right) \subset(0,1), \quad s \in \mathbb{N} .
$$

In addition, for $w_{\ell j} \in \mathbb{R}$ the final number of bound states, $N_{\ell}=(2 \ell+1) n_{\ell}$, is determined by

$$
n_{\ell}=M+m-m^{\prime}
$$

where $M$ is

$$
M=\min \left\{s \in \mathbb{N}_{0} \mid j_{\ell+1 / 2, s+1}>v_{0}\right\},
$$

and, using the functions $\varphi_{\ell}(\chi)$ and $\phi_{\ell}(\sigma)$ defined after (27),

$m=\left\{\begin{array}{lll}1 & \text { if } \quad \varphi_{\ell}\left(v_{0}\right)>\phi_{\ell}\left(0^{+}\right), \\ 0 & \text { if } \quad \varphi_{\ell}\left(v_{0}\right)<\phi_{\ell}\left(0^{+}\right) \text {or } v_{0}=j_{\ell+1 / 2, M},\end{array}\right.$ $m^{\prime}=\left\{\begin{array}{l}1 \\ 0\end{array}\right.$

Observe that the cases $\varphi_{\ell}\left(v_{0}\right)=\phi_{\ell}\left(0^{+}\right)$and $\phi_{\ell}\left(v_{0}\right)=0$ are a priori excluded from the present study. The same holds true for the possible bound states with energy below the potential well, which can arise if $\phi_{\ell}\left(v_{0}\right)<0$. We have focused on the states that are considered in meanfield nuclear models in order to facilitate the application. In addition, it is interesting to point out that the structure of the energy intervals (32) is unaffected by the $\delta^{\prime}$ interaction as long as (31) holds. Moreover, the number of bound states is mainly determined by $M$. For example, in Figure 1 we observe that this number remains the same for different values of $\beta$. The same conclusion holds for the isotope ${ }^{209} \mathrm{~Pb}$, as will be shown in the last section. This fact could eventually justify the interpretation of this $\delta^{\prime}$ interaction as an extra mean-field interaction less relevant than the spin-orbit one. 


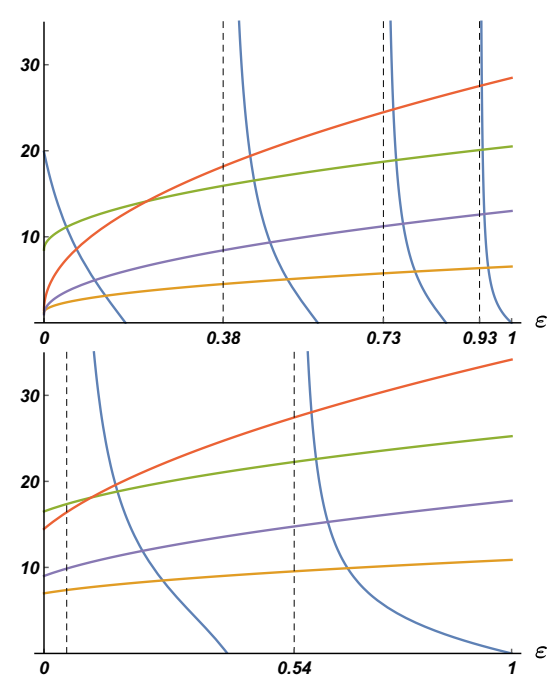

Fig. 1. Bound states from the secular equation (27): the lefthand $\varphi_{\ell}$ side in blue, the right-hand side $\phi_{\ell}$ in yellow for $\beta=$ -10 , green for $\beta=0$, red for $\beta=10$, and purple for $\beta=$ $\pm \infty$. The figure on the left corresponds to $\ell=0$, the figure on the right to $\ell=4$. In both cases, the values of the relevant parameters are chosen to be $v_{0}=12, w_{\ell j}=30$.

In theorem 1, we have assumed the existence of an upper bound for the angular momentum, $\ell_{\max }$. For spherically symmetric potentials, satisfying $\int_{0}^{\infty}|V(r)| r^{t} d r<\infty$ for $t=1,2$, the inequality

$$
n_{\ell}<\frac{1}{2 \ell+1} \int_{0}^{\infty} r\left|V_{-}(r)\right| d r, \quad V_{-}(r)=\min (\{V(r), 0\}),
$$

ensures the existence of this upper bound [46]. For the $\delta$ potential alone, the existence of $\ell_{\max }$ is guaranteed. As a matter of fact, it can be shown that a particular linear combination of $\delta$ potentials saturates the previous inequality 47. When we add the $\delta^{\prime}$ term, the argument of Bargmann [46] for the existence of $\ell_{\max }$ does not apply any more, since it is then unclear how to interpret the previous integral. Fortunately, the following result, for which the proof is given in appendix B.2, guarantees the existence of this bound in the present configuration. Furthermore, it also provides a simple expression for $\ell_{\max }$.

Theorem 2 There are no bound states with angular momentum $\ell>\ell_{\max }$, where

$$
\ell_{\max }=\max \left\{\ell \in \mathbb{N}_{0} \mid j_{\ell+1 / 2,1}<v_{0} \text { or } \varphi_{\ell}\left(v_{0}\right)>\phi_{\ell}\left(0^{+}\right)\right\} .
$$

If there exist $s_{0} \in \mathbb{N}$ and $\ell_{0} \in \mathbb{N}_{0}$ such that $v_{0}=j_{\ell_{0}+1 / 2, s_{0}}$ the second condition in the previous set can not be evaluated. Nonetheless, it is not necessary since the existence of at least one bound state for $\ell_{0}$ is guaranteed.

Concerning the ordering of bound states, we can prove an important result, that will be useful in the sequel. For spherically symmetric potentials, we know that if $E_{n \ell}$ denotes the energy of a bound state defined by the quantum numbers $n$ and $\ell$ the following inequalities hold,

$$
E_{n \ell}<E_{(n+1) \ell}<E_{(n+1)(\ell+1)}, \quad n, \ell \in \mathbb{N}_{0} .
$$

This statement can be derived for continuous potentials using Sturm's theorem analysing the spectral properties of the Hamiltonian [48. Now, we extend this result for the spherically symmetric $\delta$ - $\delta^{\prime}$ interaction we are dealing with (15), where we have to take into account an additional quantum number $j$. The proof of the following result is given in appendix B.3.

Theorem 3 If there exist bound states with relative energies $\varepsilon_{n \ell_{j}}, \varepsilon_{(n+1) \ell_{j}}, \varepsilon_{n(\ell+1)_{j}}$ for $n, \ell \in \mathbb{N}_{0}$ the following inequalities hold:

$$
\text { (a) }-\varepsilon_{n \ell_{j}}<-\varepsilon_{(n+1) \ell_{j}}, \quad \text { (b) }-\varepsilon_{n \ell_{j}}<-\varepsilon_{n(\ell+1)_{j}},
$$$$
\text { (c) }-\varepsilon_{n \ell}
$$

The second inequality only applies for $j=\ell+1 / 2$ and the third inequality for $\ell>0$.

These theorem is in agreement with the results of the nuclear shell model, reinforcing the application as a limiting case of the Woods-Saxon potential in section 6. Inequalities (a) and (b) are well known in nuclear physics when dealing with spherically symmetric potentials 49, 50, as it was already mentioned in (36). With respect to inequality (c), it is worth mentioning that the microscopic quantum description of nucleons inside the nuclei, requires a careful treatment of the orbital angular momentum with the intrinsic nucleon spin. This was connected with the longstanding problem of the inability to theoretically explain the magic numbers in atomic nuclei. Only when this interaction was included in the mean-field shell model, all experimental magic number were explained. In the course of this breakthrough it was found that, contrary to atomic electrons, the nucleon which is aligned with the orbital angular momentum is more strongly attracted. This is consistent with the previous theorem.

Lastly, a brief comment on the ground state. For a single particle Schrödinger equation it can be shown, using the variational principle, that the ground state must be a spherically symmetric zero angular momentum state [51]. For the spherical potential well, this statement can be directly proved with the secular equation (27) using the monotonicity properties of $\varphi_{\ell}(\chi(\varepsilon))$ and $\phi_{\ell}(\sigma(\varepsilon))$; in this case $\alpha=0$ and $\beta=0$ so the right-hand of equation (27) is strictly positive.

However, with the results shown, this could seem to be no longer true when we add the $\delta$ - $\delta^{\prime}$ interaction. In fact, it would be enough to include the $\delta$ potential. For example, there exists configurations with bound states for $\ell=1$ and not for $\ell=0$. Although a two particle system with strong spin-orbit coupling can end up with a non-zero angular momentum ground state [52, in the above-mentioned configurations the $\ell=0$ bound state exits. As we have already mentioned after theorem 1, an attractive $\delta$ coupling such that $\phi_{\ell}\left(v_{0}\right)$ involves a bound state with energy below $-V_{0}$, whereas we are focusing on states lying within $\left(-V_{0}, 0\right)$, see section 3.1. It is worth mentioning that the $\delta$ - $\delta^{\prime}$ interaction without the spherical well also presents bound states with angular momentum $\ell \in\left\{0,1, \ldots, \ell_{\max }\right\}$. This statement has been proved in theorem 2 of [44]. 


\subsection{Special cases $\beta= \pm 2$}

Let us go back to the matching conditions 26 . They do not apply for the exceptional values $\beta= \pm 2$. Nevertheless, there exist respective self adjoint extensions of the radial Hamiltonian for these cases [10]. They are characterized by the following $\mathrm{BC}$ at $r=R$ :

$$
\begin{array}{ll}
u_{\ell}\left(R^{+}\right)-\frac{4}{\alpha} u_{\ell}^{\prime}\left(R^{+}\right)=0, & u_{\ell}\left(R^{-}\right)=0, \quad \text { if } \quad \beta=2, \\
u_{\ell}\left(R^{-}\right)+\frac{4}{\alpha} u_{\ell}^{\prime}\left(R^{-}\right)=0, & u_{\ell}\left(R^{+}\right)=0, \quad \text { if } \quad \beta=-2 .
\end{array}
$$

These situations have already been studied, for instance in 45], where it is shown that in both cases the contact interaction becomes an opaque barrier, which means that the transmission coefficient is equal to zero. This suggests that there are only bound states, in an infinite number, plus scattering states and no resonances whatsoever. We may give an estimation of the values and number of bound states when $\beta \rightarrow \pm 2$.

First of all, taking the limit $\beta \rightarrow 2$ in 27) when $\operatorname{sgn}\left(\phi_{\ell}\left(0^{+}\right) \phi_{\ell}\left(v_{0}\right)\right)=1$ we obtain $\left|\phi_{\ell}(\sigma)\right| \rightarrow \infty$. Therefore, the acceptable values of $\chi$ in the same equation are, essentially, the zeros of $J_{\ell+1 / 2}(\chi)$. Hence, from $\chi=v_{0} \sqrt{1-\varepsilon}$, we conclude that the $i$-th bound state with relative energy $\varepsilon_{i}$ is given by

$$
\lim _{\beta \rightarrow 2} \varepsilon_{i}=1-\left(\frac{j_{\ell+1 / 2, i}}{v_{0}}\right)^{2} .
$$

Note that the first energy value, $\varepsilon_{1}$, is not reached if $\operatorname{sgn}\left(\phi_{\ell}\left(0^{+}\right)\right)=-1$. Secondly, in the limit $\beta \rightarrow-2$ equation (27) reduces to

$$
\frac{\chi J_{\ell+\frac{3}{2}}(\chi)}{J_{\ell+\frac{1}{2}}(\chi)}=\left(\ell+1+\frac{w_{\ell j}}{16}\right) .
$$

This transcendental equation is far simpler than (27) since the right-hand side is independent of $v_{0}$ and the relative energy.

\subsection{Large-parameter configurations}

The main aim of this section is to show that for certain values of the parameters $v_{0}, w_{\ell j}$ and $\beta$ remarkable simplifications in the bound state structure occur. To begin with, let us consider $v_{0} \gg 0$. Hence, using the limiting forms of the Bessel functions for large values of their arguments [53, the secular equation (27) can be approximated by

$$
\chi \cot \left(\frac{\pi \ell}{2}-\chi\right)=\frac{(\beta+2)^{2} \sigma-8 \beta(\ell+1)+w_{\ell j}}{(\beta-2)^{2}} .
$$

It is important to note that the the previous equation only differs from the zero angular momentum secular equation by the term $8 \beta(\ell+1)=8 \beta$. Consequently, for the $\delta$ potential alone and $\ell=0$, equation (27) takes the following simple form:

$$
-\chi \cot \chi=\frac{w_{\ell j}}{4}+\sigma \text {. }
$$

In this regard, we should mention that this approach is valid for low angular momentum values only. For instance, after (40) we can not conclude the existence of the maximal angular momentum $\ell_{\max }$ defined in theorem 2 .

If, in addition, we consider $\left|w_{\ell j}\right| \gg|\beta|$ so that the right-hand side of (40) is nearly independent of $\varepsilon$, the energy of the bound states can be obtained, in an approximate form, from the zeros of $\sin \left(\frac{\pi \ell}{2}-v_{0} \sqrt{1-\varepsilon}\right)$, i.e.,

$$
\varepsilon_{n} \simeq 1-\left(\frac{\pi(\ell-2 n)}{2 v_{0}}\right)^{2} \in(0,1), \quad n \in \mathbb{Z} .
$$

Then, we may estimate the number of bound states for a given value $\ell$ of the angular momentum as

$$
N_{\ell}=n_{\ell}(2 \ell+1) \quad \text { with } \quad n_{\ell} \simeq\left\lfloor\frac{\pi \ell+2 v_{0}}{2 \pi}\right\rfloor \simeq\left\lfloor\frac{v_{0}}{\pi}\right\rfloor
$$

where the number of negative energy values $n_{\ell}$ has been obtained from (42), under the condition $\varepsilon_{n}>0$.

Finally, irrespective of the previous considerations, we analyse a system characterized by a very strong $\delta^{\prime}$ interaction, that is to say, we take the limit $|\beta| \rightarrow \infty$ in the secular equation (27). As can be easily checked, this situation is equivalent to the non-existence of the $\delta$ - $\delta^{\prime}$ interaction, i.e., $\alpha=\beta=0$. For this particular example, the matching conditions $(26)$ impose the continuity of the radial function and its first derivative. The resulting secular equation matches with the one found for the finite three-dimensional spherical potential well, usually derived imposing the continuity of the logarithm derivative of the radial function at $R$ [48].

\section{Resonances}

Solvable or quasi-solvable models usually have, in addition to bound states, resonances (unstable or quasi-stable quantum states) and possibly anti-bound states. The study of resonance models is necessary because most of the known quantum states are unstable. For example, single-particle resonances appear in the dripline of light nuclei, such as ${ }^{5} \mathrm{He},{ }^{8} \mathrm{~B}$, and ${ }^{10} \mathrm{Li}$. Resonance models give a qualitative account for resonance behaviour and, therefore, may give a good insight into the quantum properties of unstable states. In this paper, we are assuming that resonances appear in resonance scattering, which is produced by a Hamiltonian pair $\left\{H_{0}, H=H_{0}+V\right\}$. Thus, a resonance arises when the incoming particle stays in the region where the potential acts a much longer time than the one it would have stayed if the potential had not existed.

There are several definitions of resonances based on either physical or mathematical notions, which are not always equivalent. Because of the kind of model presented here, we are using the concept of resonance as given in mathematical terms. There are essentially two approaches, either we define resonances as poles of analytic continuations of a reduced resolvent of the total Hamiltonian [56], or as poles of an analytic continuation of the $S$-matrix in the momentum representation (or equivalently in the 
energy representation). Here, we shall adopt the second point of view.

Under some general conditions based on causality principles [57, the $S$ matrix in momentum representation, denoted by $S(k)$, admits an analytic continuation to a meromorphic function of the complex variable $k$ on the whole complex plane. It is meromorphic because $S(k)$ has poles, which may be classified in three types:

- Simple poles on the positive half of the imaginary axis that correspond to bound states.

- Simple poles in the negative half of the imaginary axis, which represent the presence of the antibound (virtual) states.

- Pair of poles on the lower half plane, symmetrically located with respect to the imaginary axis, each of these pairs representing one resonance [57,58].

Although the order of resonance poles may be in principle arbitrary (both poles of each pair must have the same multiplicity), in general they are simple. This result emerges from our particular model.

If we go from the momentum to the energy representation, $E=\hbar^{2} k^{2} /(2 \mu)$, poles for each resonance pair become two conjugate complex numbers of the form $z_{R}=$ $E_{R}-i \Gamma / 2$ and $z_{R}^{*}=E_{R}+i \Gamma / 2$, with $\Gamma>0$. Here, $E_{R}$ represents the resonance energy, usually $E_{R}>0$, and $\Gamma$ the inverse of the half life. After this, one may understand that in the momentum representation, the closer a resonance pole is to the real axis, the higher is its mean life.

Without further ado, let us study the resonances in the present case. In section 3.2 , we have written the wave equation outside the nucleus as a linear combination of modified Bessel functions of first and second kind. The requirement of square integrability, needed to characterize bound states, forced us to drop the contribution of the Bessel function of first kind and just keep the Bessel function of second kind. Resonance state functions, also called Gamow functions, are not square integrable so we can simply solve Schrödinger equation at $r>R$ for $E>0$. This leads to a solution analogous to (18) in terms of Bessel and Neumann functions. Nevertheless, for reasons that will be evident below, it is convenient to write this solution in terms of the Hänkel functions as

$u_{\ell}(r)=\sqrt{\kappa r}\left(C_{\ell} H_{\ell+\frac{1}{2}}^{(1)}(\kappa r)+D_{\ell} H_{\ell+\frac{1}{2}}^{(2)}(\kappa r)\right), \quad \kappa=\frac{\sqrt{2 \mu E}}{\hbar}$

where $C_{\ell}$ and $D_{\ell}$ are independent of $r$, although they depend on $\kappa$. This expression is valid for $r>R$. The superscripts distinguish between the Hänkel functions of first and second kind. These functions present the following asymptotic behaviour 53 for large values of $r$ :

$H_{\ell+\frac{1}{2}}^{(1)}(\kappa r) \sim \sqrt{\frac{2}{\pi \kappa r}} e^{i(\kappa r-(\ell+1) \pi / 2)}, \quad-\pi<\arg z<2 \pi$,

Consequently, $H_{\ell+1 / 2}^{(1)}(\kappa r)$ can be interpreted as an outgoing wave function, while $H_{\ell+1 / 2}^{(2)}(\kappa r)$ as an incoming wave function. Resonances are given by the so called purely outgoing boundary condition, which states that only the outgoing wave function survives. This is satisfied if, and only if, $D_{\ell}=0$ in (44). At first look, this may resemble to the requirement $C_{\ell}=0$ for $(23)$, although the situation here has a completely different origin. Furthermore, the transcendental equation $D_{\ell}(\kappa)=0$ gives us the poles of the $S$-matrix, which are the resonant poles.

In order to obtain these poles in the momentum representation and, after that, proceed with the construction of the resonance Gamow wave functions, we also use the matching condition between the outgoing function and the wave function inside the potential well, previously calculated in section 3.1. This gives the following transcendental equation in $k$

$$
\begin{aligned}
H_{\ell+\frac{1}{2}}^{(1)}(R \kappa)[ & 8(\alpha R-\beta) J_{\ell+\frac{1}{2}}(R \gamma)-(\beta-2)^{2} R \gamma J_{\ell+\frac{3}{2}}(R \gamma)+(\beta-2)^{2} \\
& +(\beta+2)^{2} \kappa R J_{\ell+\frac{1}{2}}(R \gamma) H_{\ell+\frac{3}{2}}^{(1)}(R \kappa)-(\beta+2)^{2} \kappa R J_{\ell+\frac{1}{2}}(R
\end{aligned}
$$

The solutions of (45) should be classified in three categories, as previously explained. If we set $\ell=0$, the situation simplifies enormously. In fact, 45 becomes

$$
\frac{\tan (\gamma R)}{\gamma R}=-\frac{i(\beta-2)^{2}}{(\beta+2)^{2} \kappa R+4 i \alpha R}
$$

When we choose $\beta=0$ (absence of the term in $\delta^{\prime}$ ), $V_{0}=0$ $(\gamma=\kappa)$ and $\ell=0$, we recover well known results of onedimensional systems [2,14]. Since the resonant poles are complex solutions in the momentum representation, let us use the following notation:

$$
k_{1}+i k_{2}=: R \kappa, \quad R \gamma=\sqrt{v_{0}^{2}+\left(k_{1}+i k_{2}\right)^{2}},
$$

so that 46 may be written as

$$
F\left(k_{1}, k_{2}\right):=\frac{\tan \sqrt{v_{0}^{2}+\left(k_{1}+i k_{2}\right)^{2}}}{\sqrt{v_{0}^{2}+\left(k_{1}+i k_{2}\right)^{2}}}+\frac{i(\beta-2)^{2}}{(\beta+2)^{2}\left(k_{1}+i k_{2}\right)+i w_{\ell j}} \neq 4
$$

Denoting the real and imaginary parts of the complex number $z$ by $\operatorname{Re} z$ and $\operatorname{Im} z$, respectively, a simple analysis on (48) shows that

$$
\operatorname{Re} F\left(-k_{1}, k_{2}\right)=\operatorname{Re} F\left(k_{1}, k_{2}\right) \quad \text { and } \quad-\operatorname{Im} F\left(-k_{1}, k_{2}\right)=\operatorname{Im} F\left(k_{1},\right.
$$

We Brse that (49) implies that the curves in the plane $\left(k_{1}, k_{2}\right)$ given by $\operatorname{Re} F\left(k_{1}, k_{2}\right)=0$ and $\operatorname{Im} F\left(k_{1}, k_{2}\right)=0$ are symmetric with respect to the imaginary axis $k_{1}=0$. The behaviour of the solutions of equation (48) is shown in Figure 2, Except for the intersections of these two curves in the negative imaginary semi-axis, antibound states, the ones in the lower half plane give the resonance poles.

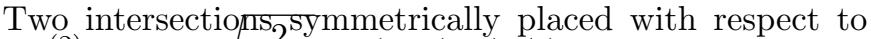

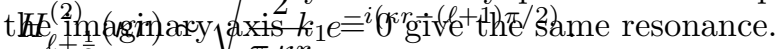

${ }^{\ell} \frac{1}{2}$ also obser $\frac{\pi}{2} r_{\text {the }}$ existence of a bound state in the positive imaginary axis $k_{2}>0$. These results are consistent with those obtained for bound states earlier in this paper; when we set $k_{1}=0$ and $k_{2}>0$ in 48 we recover the secular equation for $\ell=0$, see 40 and the comment underneath. It is noteworthy that there is an infinite number of resonances which lie on the lower half plane without 


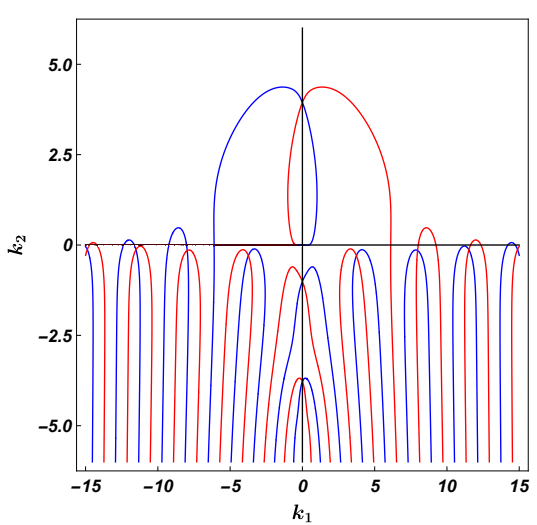

Fig. 2. In blue $\operatorname{Re} F\left(k_{1}, k_{2}\right)=0$ and in $\operatorname{red} \operatorname{Im} F\left(k_{1}, k_{2}\right)=0$, from 45 for $\ell=0$. Bound states and resonances correspond to intersection of red and blue curves. The relevant parameters are chosen to be $v_{0}=5, w_{\ell j}=10$ and $\beta=1$.

the real axis $k_{2}=0$. In fact, for $k_{2}=0$, the imaginary part of 48 is given by

$$
\frac{\left(\beta^{2}-4\right)^{2} k_{1}}{(\beta+2)^{4} k_{1}^{2}+w_{\ell j}^{2}}=0,
$$

which implies $k_{1}=0$, so that all intersections should coincide on the origin, which is obviously not the case. This is important, since as a consequence of reasonable causality conditions [57] resonance poles should lie on the lower half plane in the momentum representation.

Equation 45 for $\ell \neq 0$ does not admit the kind of simplification yielding to 46 . Nevertheless, it is still possible to give an estimation of the location of the first few resonances in the $k$ plane as well as some antibound states. Our results are depicted in Figure 3, where the cases $\ell=1,2,3,4$ are considered. Resonance poles are located at the intersections of red and blue curves right below the real axis. Antibound states poles are located at the intersections of blue and red curves on the negative imaginary axis. The structure of the solutions is similar to the case $\ell=0$.

\section{Neutron energy levels of ${ }^{133} \mathrm{Sn}$ and ${ }^{209} \mathrm{~Pb}$}

The purpose of this final section is to briefly discuss the general results previously obtained in the context of a realistic physical situation. To begin with, we are going to use the program know as the Gamow code for some of our estimations. It is a numerical program which gives the energy of bound states for the Woods-Saxon potential (9) and it is quite useful for various reasons. First of all, it serves to estimate how good the approximation $a \rightarrow 0^{+}$is. In addition, the Gamow code permits a comparison with the experimental results. Note that this code supplies more values than the current experimental data. We should also remark that our goal is to show that our results are qualitatively reasonable for low-lying bound states and that we do not intend to get a numerical fit with good precision, which is beyond the purpose of the model.
In Table 2, we compare the Gamow code 32 and experimental energies, taken from the Database of the National Nuclear Data Center Brookhaven National Laboratory [54 and [55, for the isotope ${ }^{209} \mathrm{~Pb}$. With this comparison we ascertain that the program we are using to test our model fits with the available experimental data. The relevant parameters describing the lowest experimental energy states are $V_{0}=44.4 \mathrm{MeV}, V_{\text {so }}=16.5 \mathrm{MeV} \mathrm{fm}$, $r_{0}=1.27 \mathrm{fm}(R=7.525 \mathrm{fm}), a=0.7 \mathrm{fm}$, and $2 \mu / \hbar^{2}=$ $0.0480 \mathrm{MeV}^{-1} \mathrm{fm}^{-2}$ [54. For the present configuration $v_{0}=10.98, w_{\ell j}=-23.83 \xi_{\ell j}$.

Table 2. Comparison of the numerical (Gamow code) energy levels $(\mathrm{MeV})$ in ${ }^{209} \mathrm{~Pb}$ with the experimental ones, using the physical parameters mentioned in the text.

\begin{tabular}{llc}
\hline State $\left(n \ell_{j}\right)$ & Gamow & $E_{\exp }$ \\
\hline $1 g_{9 / 2}$ & -3.93 & -3.94 \\
$2 p_{1 / 2}$ & -7.41 & -6.73 \\
$2 p_{3 / 2}$ & -8.35 & -7.62 \\
$0 i_{11 / 2}$ & -2.80 & -3.16 \\
$2 d_{5 / 2}$ & -2.07 & -2.37 \\
$0 j_{15 / 2}$ & -1.88 & -2.51 \\
\hline
\end{tabular}

Focusing on the results that emerge from the singular Hamiltonian (15), we begin comparing the energy levels for some states of the nucleus ${ }^{133} \mathrm{Sn}$ achieved using the square well plus the $\delta$ potential alone, $\beta=0$. The energy values of the $\delta-\delta^{\prime}$ model $\left(\delta-\delta^{\prime} \mathrm{M}\right)$ are obtained through (27), where the numerical values of the physical parameters are $V_{0}=39.5 \mathrm{MeV}, V_{\text {so }}=15.5 \mathrm{MeV} \mathrm{fm}, r_{0}=1.27 \mathrm{fm}$, $2 \mu / \hbar^{2}=0.0479 \mathrm{MeV}^{-1} \mathrm{fm}^{-2}, R=6.47 \mathrm{fm}$ and $V_{\mathrm{q}}=0$ [54], which set $v_{0}=8.89, w_{\ell j}=-19.20 \xi_{\ell j}$, see (29). Some results are shown in Table 3 , where we can see that the inequalities of (37) are always satisfied. For these low-lying bound states we obtain a quantitatively fair approximation. For this simulation we have taken $a=0.05 \mathrm{fm}$.

Table 3. Neutron energy levels $(\mathrm{MeV})$ for $\ell=0$ and $\ell=1$ in the nucleus ${ }^{133} \mathrm{Sn}$, using the physical parameters mentioned in the text.

\begin{tabular}{lll}
\hline State $\left(n \ell_{j}\right)$ & Gamow & $\delta-\delta^{\prime} \mathrm{M}$ \\
\hline $0 s_{1 / 2}$ & -35.52 & -35.53 \\
$1 s_{1 / 2}$ & -23.81 & -23.83 \\
$2 s_{1 / 2}$ & -5.56 & -5.59 \\
$-\frac{-}{0}-------$ & ---- \\
$0 p_{1 / 2}$ & -31.37 & -30.79 \\
$1 p_{1 / 2}$ & -15.95 & -14.20 \\
$0 p_{3 / 2}$ & -31.42 & -31.95 \\
$1 p_{3 / 2}$ & -16.08 & -17.46 \\
\hline
\end{tabular}

Now we add the $\delta^{\prime}$ interaction in the nucleus ${ }^{209} \mathrm{~Pb}$. In Figure 4, we use the parameters given above for $\ell=0$ and $\ell=1$, where the energy of the bound states is given in Table 4 . We also compare these results with those obtained with the Gamow code. We have chosen $\beta=0$ and $\beta=1$, 
which corresponds to $V_{\mathrm{q}}=0$ and $V_{\mathrm{q}}=-20.83 \mathrm{MeV} \mathrm{fm}^{2}$, respectively. The numerical approximation of the square well and singular potentials in Table 4 are simulated taking $a=0.01 \mathrm{fm}$. From Table 4 , we observe that there are three bound states for both values $\beta=0$ and $\beta=1$. The same number of bound states is obtained when we consider the $\delta$ - $\delta^{\prime}$ M, see Figure 4. In Table 4 we have observed some discrepancies between the results obtained with our formalism and the numerical calculations obtained when considering the $\delta^{\prime}$ interaction as a limit of odd functions in the Gamow code. This is to be expected and the origin lies in the different definitions of the $\delta^{\prime}$ interaction explained at the end of section 2. Nevertheless, our intention is to show how these differences vary with the quantum numbers $(n, \ell, j)$. Indeed, in the data of Table 4 we can verify that the inequalities of (37) are always satisfied when the $\delta^{\prime}$ term is added.

Table 4. Comparison of the neutron energy levels $(\mathrm{MeV})$ in the core of ${ }^{208} \mathrm{~Pb}$ between the numerical Gamow code results [32] versus our model $\left(\delta-\delta^{\prime} \mathrm{M}\right)$ results, using the physical parameters mentioned in the text. Calculations are done for $\beta=0$ (second and third columns) and $\beta=1$ (fourth and fifth columns).

\begin{tabular}{|c|c|c|c|c|}
\hline \multirow{2}{*}{ State } & \multicolumn{2}{|c|}{$\beta=0$} & \multicolumn{2}{|c|}{$\beta=1$} \\
\hline & Gamow & $\delta-\delta^{\prime} \mathrm{M}$ & Gamow & $\delta-\delta^{\prime} \mathrm{M}$ \\
\hline $0 s_{1 / 2}$ & 5 & 1.36 & 0.97 & -40.84 \\
\hline $1 s_{1 / 2}$ & -32.27 & -32.31 & -31.11 & -30.23 \\
\hline $2 s_{1 / 2}$ & -17.53 & -17.61 & -18.11 & -12.91 \\
\hline $0 p_{1 / 2}$ & $-38 . \overline{0} 8$ & -37.80 & $-3 \overline{7} . \overline{3} 4$ & -37.10 \\
\hline $1 p_{1 / 2}$ & -25.91 & -25.04 & -24.44 & -22.90 \\
\hline $2 p_{1 / 2}$ & -8.47 & -6.67 & -11.20 & -2.38 \\
\hline$\overline{0} p_{3 / 2}$ & $-38 . \overline{2} 1$ & -38.54 & $-3 \overline{7} . \overline{4} 8$ & $-37 . \overline{15}$ \\
\hline $1 p_{3 / 2}$ & -26.29 & -27.18 & -25.30 & -23.16 \\
\hline $2 p_{3 / 2}$ & -9.17 & -10.63 & -13.30 & -4.31 \\
\hline
\end{tabular}

\section{Concluding remarks}

We have studied a spherical well plus a linear combination of a Dirac delta and a local $\delta^{\prime}$ interaction, both located at the well edge. Due to spherical symmetry, the problem reduces to a one-dimensional one, by means of the radial Schrödinger equation. This contact potential has been defined by using appropriate matching conditions satisfied by the radial wave functions. In particular, we have obtained general and precise properties concerning the number and behaviour of bound states. These are summarized in the three theorems of section 4. Note that due to the singular character of the studied interaction, general results applicable to well-behaved spherical potentials can not be used. Nevertheless, we have been able to extend some of them to our case. In addition, we have even obtained some precise analytical expressions, as for instance, we not only guarantee the existence of $\ell_{\max }$, we provide a specific expression. We have also presented the simplifications in the bound states structure for certain configurations of the parameters of the model.

We have found that the dependence of bound states with the coefficient $\alpha$ of the $\delta$ potential is stronger than the dependence on the coefficient $\beta$ of the $\delta^{\prime}$ interaction. There are, nevertheless, some exceptions, the most interesting occurs when $\beta$ reaches two critical points, yielding to the appearance of Robin or Dirichlet BC.

This model has also antibound states and resonances. These are characterized by the existence of poles in the analytic continuation, $S(k)$, of the $S$-matrix in the moment representation. These poles may also be obtained using the so-called purely outgoing boundary conditions, which are determined by equating to zero the coefficient of the asymptotic form of the incoming wave. This coefficient depends on the momentum $k$, which gives a transcendental equation, for which the solutions are the poles of $S(k)$. Exact and numerical values for resonances, bound and antibound states can be obtained for all values of the orbital angular momentum, although the case $\ell=0$ is by far the simplest.

In the last section we have used this configuration to approximately describe the extra neutron energy levels of a double magic nucleus with spin-orbit interaction plus an extra mean-field interaction, testing the numerical results with the nuclei ${ }^{209} \mathrm{~Pb}$ and ${ }^{133} \mathrm{Sn}$. We have shown that the Hamiltonian (14), inspired by the Woods-Saxon potential after the limit $a \rightarrow 0^{+}$, gives a good approximation for the low-lying bound states. The $\delta$ term gives the nuclear spinorbit contribution. The aim of the additional interaction, given by the $\delta^{\prime}$ interaction, is providing a correction such that the results of the proposed model better fit to the experimental data, in particular for states like the ones shown in Table 2. This simplified model could be used to gain insight into the neutron energy levels since the main advantage over the Woods-Saxon potential is that we can solve exactly the eigenfunction equation, obtaining analytic properties of the spectrum using well-known features of Bessel functions. In any case, our goal is to describe properties in a qualitative manner, we do not expect to get a numerical fit with good precision.

Along our discussion, we have mentioned that there are two possible choices of the $\delta^{\prime}$ interaction. As pointed out before, we have chosen the only one which is compatible, the resulting Hamiltonian is self adjoint, with the $\delta$ interaction supported at the same point, the so called local $\delta^{\prime}$ interaction. We have obtained some numerical results, which slightly deviate from those obtained using the regular mean-field potential. The reason is that the limit $a \rightarrow 0^{+}$in 13 leads to a $\delta^{\prime}$ potential which does not give a self adjoint version of the Hamiltonian.

As a final remark, we may explore similar approximations with potentials of another type which may be also interesting in nuclear systems, in the nearest future. One possibility is to replace the three dimensional Wood-Saxon potential by the three dimensional Scarf II potential as studied by Lévai and coworkers 64. 


\section{Acknowledgements}

This work was supported by Consejo Nacional de Investigaciones Científicas y Técnicas PIP-625 (CONICET, Argentina), the Spanish MINECO (MTM2014-57129-C2-1P), Junta de Castilla y León and FEDER projects (BU229P18 and VA137G18). C.R. is grateful to MINECO for the FPU fellowships programme (FPU17/01475).

\section{Appendix A On the self adjointness of the Hamiltonian}

The goal of the present appendix is a discussion on the self adjointness of the radial Hamiltonian (15). Setting the appropriate units such that $\hbar=1$ and $2 \mu=1$ this Hamiltonian may be written as

$H=-\frac{d^{2}}{d r^{2}}+[\theta(r-R)-1] V_{0}+a \delta(r-R)+b \delta^{\prime}(r-R)+\frac{\ell(\ell+1)}{r^{2}}$,

$\ell \in \mathbb{N}_{0}$. Let us split it into $H=H_{\ell}+V(r)$, where

$$
H_{\ell}=-\frac{d^{2}}{d r^{2}}+\frac{\ell(\ell+1)}{r^{2}} .
$$

For the sake of clarity, we first study $H_{\ell=0}$, which reduces to the one-dimensional Laplace operator in a given domain.

\section{A.1 Zero angular momentum}

We have to find a domain for $H_{0}$, which must be a subspace of $L^{2}[0, \infty)$. This domain must include all square integrable absolutely continuous functions, $f(r)$, with absolutely continuous derivative and square integrable second derivative. Thus,

$$
\int_{0}^{\infty}\left\{|f(r)|^{2}+\left|f^{\prime \prime}(r)\right|^{2}\right\} d r<\infty
$$

The boundary conditions at the origin should be specified in such a way that $H_{0}$ is Hermitian on its domain. In consequence, for any $f(r), g(r)$ in the domain of $H_{0}$,

$$
\begin{aligned}
\left\langle h(r) \mid H_{0} f(r)\right\rangle & =-\int_{0}^{\infty} h^{*}(r) f^{\prime \prime}(r) d r=h^{*}(0) f^{\prime}(0)-h^{\prime *}(0) \\
& =h^{*}(0) f^{\prime}(0)-h^{\prime *}(0) f(0)+\left\langle H_{0} h(r) \mid f(r)\right\rangle .
\end{aligned}
$$

Then, $H_{0}$ is Hermitian in the given domain if, and only if, $h^{*}(0) f^{\prime}(0)-h^{\prime *}(0) f(0)=0$, which happens if, and only if, $f(0)=c f^{\prime}(0)$ for any function $f(r)$ in this domain, where $c$ is an arbitrary real constant. For $c=0$, we have that $f(0)=0$ with $f^{\prime}(0)$ arbitrary. Since $c^{-1} f(0)=f^{\prime}(0)$, another possible choice is $f^{\prime}(0)=0$ with $f(0)$ arbitrary. Here, we may say that $c=\infty$. All these possible choices select a domain, $\mathcal{D}$, in which $H_{0}$ is self adjoint. We select any one of them.
After selecting a value of $c \in \mathbb{R} \cup\{\infty\}$, let us consider a subspace of $\mathcal{D}$, denoted by $\mathcal{D}\left(H_{0}\right)$. By definition, $f(r) \in \mathcal{D}\left(H_{0}\right)$ if, and only if, $f(R)=f^{\prime}(R)=0$. Choosing $\mathcal{D}\left(H_{0}\right)$ as the domain of $H_{0}$, we see that $H_{0}$ is symmetric (Hermitian), although not self adjoint, having deficiency indices $(2,2)$.

In order to prove this latter statement, let us recall that the domain of the adjoint $H_{0}^{\dagger}$ is determined by

$$
\mathcal{D}\left(H_{0}^{\dagger}\right)=\left\{h(r) \in L^{2}[0, \infty) ; \exists g(r) \in L^{2}[0, \infty) ;\left\langle h(r) \mid H_{0} f(r)\right\rangle=\langle g(r\right.
$$

for all $f(r)$ in $\mathcal{D}\left(H_{0}\right)$. To obtain a basis of the deficiency subspaces [59], we have to solve the equations $h^{\prime \prime}(r)=$ $\pm i h(r)$, where the solutions must be in $\mathcal{D}\left(H_{0}^{\dagger}\right)$. Let us choose the sign plus first. We obtain two linearly independent solutions, which are:

$$
h_{1}(r)=\left\{\begin{array}{cc}
C e^{-\frac{\sqrt{2}}{2} r} e^{-i \frac{\sqrt{2}}{2} r}, & r>R, \\
0, & r<R,
\end{array} \quad h_{2}(r)=\left\{\begin{array}{l}
A e^{-\frac{\sqrt{2}}{2} r} e^{-i \frac{\sqrt{2}}{2} r}
\end{array}\right.\right.
$$

where $A$ and $C$ are arbitrary constants. The linear independence of these two functions is obvious, so that they are a basis for the deficiency subspace corresponding to the plus sign. Similar analysis can be performed for the minus sign. This proves that the deficiency indices for $H_{0}$ with domain $\mathcal{D}\left(H_{0}\right)$ are precisely $(2,2)$. In this circumstance, $H_{0}$ admits an infinite number of self adjoint extensions labelled by four independent real parameters. Domains for these self adjoint extensions are determined by matching conditions at the point $r=R$ as usual [10, where the exceptional cases $\beta= \pm 2$ are also included. The choice of the matching conditions 26 gives a two parametric family of self adjoint extensions, which proves the self adjointness of

$$
H_{r}=-\frac{d^{2}}{d r^{2}}+a \delta(r-R)+b \delta^{\prime}(r-R),
$$

which is A.1 with $\ell=0$ and without the term $V_{0}[\theta(r-$ $R)-1$. As we will explain at the end of the present appendix, adding this term to the potential does not change the self adjointness.

\section{A.2 Higher angular momentum}

For $\ell \geq 1$ do not need to impose conditions at the ori-

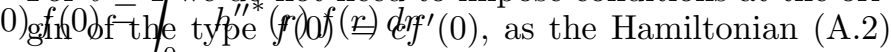
is essentially self adjoint when its domain is given by the Schwartz space suppo(rted) on $\mathbb{R}^{+}:=[0, \infty)$. For these functions $f(0)=f^{\prime}(0)=0$, so that $h^{*}(0) f^{\prime}(0)-h^{\prime *}(0) f(0)$ is automatically zero. Then, we define $\mathcal{D}\left(H_{\ell}\right), \ell \neq 0$, to be the space of functions $f(r) \in L^{2}[0, \infty)$ satisfying the following conditions [4]:

1. $f(r)$ and $f^{\prime}(r)$ are absolutely continuous.

2. $-f^{\prime \prime}(r)+\left[\ell(\ell+1) / r^{2}\right] f(r)$ is square integrable, i.e., it belongs to $L^{2}[0, \infty)$.

3. $f(0)=0$.

4. $f(R)=f^{\prime}(R)=0$. 
In order to obtain the deficiency subspaces for $H_{\ell}$, we have to find the square integrable solutions of the following pair of differential equations:

$$
h^{\prime \prime}(r)-\frac{\ell(\ell+1)}{r^{2}} h(r) \mp i h(r)=0 .
$$

For the minus sign in A.7), the general solution is given by 43 (p. 478):

$$
u(r)=A r^{1 / 2} J_{\ell+1 / 2}(r \sqrt{i})+B r^{1 / 2} Y_{\ell+1 / 2}(r \sqrt{i}),
$$

where $J_{\ell+1 / 2}$ and $Y_{\ell+1 / 2}$ are the Bessel and Neumann functions [60], respectively. Asymptotic properties of these functions show that 60,43 :

$r^{1 / 2} J_{\ell+1 / 2}(r \sqrt{i}) \notin L^{2}[1, \infty), \quad r^{1 / 2} J_{\ell+1 / 2}(r \sqrt{i}) \in L^{2}[0,1]$, $r^{1 / 2} Y_{\ell+1 / 2}(r \sqrt{i}) \in L^{2}[1, \infty), \quad r^{1 / 2} Y_{\ell+1 / 2}(r \sqrt{i}) \notin L^{2}[0,1]$.

Therefore, the basis for the deficiency subspace with minus sign in A.7 is given by the following pair of functions:

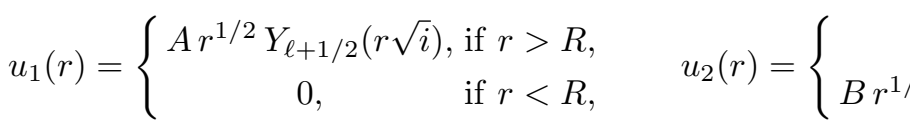$$
B
$$

where $A$ and $B$ are constants. A similar result can be obtained for the plus sign in (A.7), so that the deficiency indices for $H_{\ell}$ with $\ell \neq 0$ are $(2,2)$. Self adjoint extensions are obtained by suitable matching conditions at $r=R$ and depend on four real parameters. Again, the choice of matching conditions (26), where the exceptional cases $\beta=$ \pm 2 are included, determines a self adjoint Hamiltonian of the form,

$H_{r}:=-\frac{d^{2}}{d r^{2}}+a \delta(r-R)+b \delta^{\prime}(r-R)+\frac{\ell(\ell+1)}{r^{2}}, \quad \ell \neq 0$,

which is A.1 without the term $V_{0}[\theta(r-R)-1]$. Adding this term does not change anything in both cases $(\ell=0$ and $\ell \neq 0$ ). Once we have determined the domains for which A.6 and A.9 are self adjoint, since the term $V_{0}[\theta(r-R)-1]$ is bounded and Hermitian, it is self adjoint. Now, the Kato-Rellich theorem [59] says that if $H_{r}$ is self adjoint, so is $H_{r}+[\theta(r-R)-1] V_{0}$. We conclude that it is possible to determine domains such that A.1 is self adjoint for all values $\ell \in \mathbb{N}_{0}$.

\section{Appendix B Proofs of theorems 1, 2 and 3}

\section{B.1 Proof of theorem 1}

In the first place, we show that the right-hand side of (27) is positive and strictly growing as a function of the relative energy. As will be proved in theorem 2, there exists an upper bound for the angular momentum, $\ell_{\max }$ hence the term $8 \beta(\ell+1)$ is always finite. From Theorem 6 in 61 there exits the following bounds for the following ratio of modified Bessel functions:

$$
\sqrt{\sigma^{2}+\ell^{2}}+\ell+1 \leq \frac{\sigma K_{\ell+3 / 2}(\sigma)}{K_{\ell+1 / 2}(\sigma)}<\sqrt{\sigma^{2}+(\ell+1)^{2}}+\ell+1 .
$$

Now we can use the first inequality of B.1 together with (31) to derive:

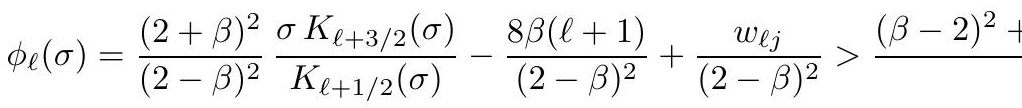

In addition, using the Turan-type inequalities given in 62], we can prove the following relation:

$$
\frac{d \phi_{\ell}(\sigma)}{d \sigma}=\frac{\sigma K_{\ell-1 / 2}(\sigma) K_{\ell+3 / 2}(\sigma)-\sigma K_{\ell+1 / 2}^{2}(\sigma)}{K_{\ell+1 / 2}^{2}(\sigma)}>0
$$

This shows that $\phi_{\ell}(\sigma)$ is a strictly growing positive function on the variable $\sigma$ and, due to the definition of $\sigma(\varepsilon)$ (28), on $\varepsilon$.

On the other hand, if we show that $\varphi_{\ell}(\chi)$, the lefthand side of (27), is one to one and onto as a function between the follfowing Rintervals:

${ }^{2} \psi_{\ell+\mathcal{X}) 2:}\left(\left(\gamma_{\ell} / \bar{i}\right)_{3 / 2, s-1}, j_{\ell+1 / 2, s}^{R}\right) \subset\left(0, v_{0}\right) \mapsto(0, \infty), s \in \mathbb{N}$,

we guarantee the unique existence of the bound state $\varepsilon_{s}$ in (32). Thus, it will be enough to demonstrate that $\varphi_{\ell}(\chi)$ is strictly monotonic on $\chi$ and that it covers the whole $(0, \infty)$ as $\chi \in\left(j_{\ell+3 / 2, s-1}, j_{\ell+1 / 2, s}\right)$. In fact, the first derivative of $\varphi_{\ell}(\chi)$ meets

$$
\frac{d \varphi_{\ell}(\chi)}{d \chi}=\chi \frac{J_{\ell+1 / 2}^{2}(\chi)-J_{\ell-1 / 2}(\chi) J_{\ell+3 / 2}(\chi)}{J_{\ell+1 / 2}^{2}(\chi)}>0
$$

where the equality follows from standard properties of the Bessel functions 53 and the second relation from the Turan-type inequalities [62,44]:

$$
J_{n}^{2}(\chi)-J_{n-1}(\chi) J_{n+1}(\chi)>\frac{J_{n}^{2}(\chi)}{n+1}>0
$$

where $\chi$ is real and $n>0$, with $n=\ell+1 / 2$. Finally, in the given intervals, the function $\varphi_{\ell}(\chi)$ is positive and

$$
\lim _{\chi \rightarrow j_{\ell+3 / 2, s-1}} \varphi_{\ell}(\chi)=\varphi_{\ell}\left(j_{\ell+3 / 2, s-1}\right)=0, \quad \lim _{\chi \rightarrow j_{\ell+1 / 2, s}^{-}} \varphi_{\ell}(\chi)=(\mathrm{B} .5)
$$

Now we focus on the second part of the theorem concerning the number of bound states (33). The key feature is the existence of an integer $s_{0}$ for which

$$
\left(j_{\ell+3 / 2, s-1}, j_{\ell+1 / 2, s}\right) \cap\left(0, v_{0}\right)=\emptyset, \forall s \geq s_{0} .
$$

Let us examine this in greater detail. The largest integer $M$ for which $\left(j_{\ell+3 / 2, M-1}, j_{\ell+1 / 2, M}\right) \subset\left(0, v_{0}\right)$ still holds is obviously given by

$$
j_{\ell+1 / 2, M}<v_{0}, \quad j_{\ell+1 / 2, M+1}>v_{0} .
$$


Since $\varphi_{\ell}(\chi(\varepsilon))$ is strictly decreasing and $\phi_{\ell}(\sigma(\varepsilon))$ strictly $\mathbf{B . 3}$ Proof of theorem 3 increasing as functions of $\varepsilon$, the condition

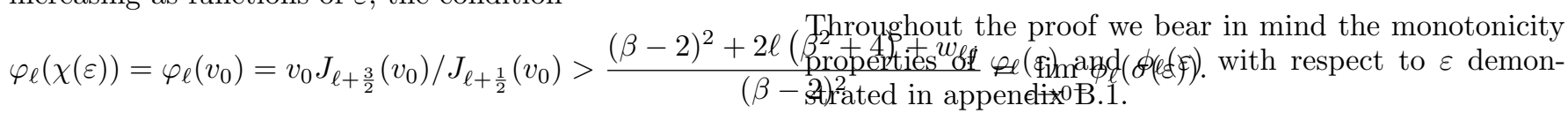
implies the existence of an additional bound state whose energy is the closest to $\varepsilon=0$. In the particular case $v_{0}=$ $j_{\ell+1 / 2, M}$ no additional bound state should be added to $M$. Independently, if

$$
\lim _{\varepsilon \rightarrow 1^{-}} \varphi_{\ell}(\chi(\varepsilon))=0>\lim _{\varepsilon \rightarrow 1^{-}} \phi_{\ell}(\sigma(\varepsilon))
$$

no bound state satisfying $\varepsilon \in\left(1-j_{\ell+1 / 2,1}^{2} / v_{0}^{2}, 1\right)$ appears. Only if $j_{\ell+1 / 2,1}>v_{0}$ the functions $m$ and $m^{\prime}$ defined in the present theorem are not independent. Nevertheless, a bound state with relative energy $\varepsilon \in(0,1)$ appears if, and only if, $\varphi_{\ell}\left(v_{0}\right)>\lim _{\varepsilon \rightarrow 0^{+}} \phi_{\ell}(\sigma)$ and $0<\lim _{\varepsilon \rightarrow 1^{-}} \phi_{\ell}(\sigma)$ so that $n_{\ell}$ is also given by (33) and the proof is concluded.

\section{B.2 Proof of theorem 2}

For this proof we use the number of bound states given in equation (33) of theorem 1 in order to obtain

$$
n_{\ell}=0 \quad \forall \ell>\ell_{\max } .
$$

Note that in the derivation of (33), we have not used the assumption of the existence of $\ell_{\max }$ that appears at the beginning of appendix B.1 so there is no circular reasoning. Due to the properties of the zeros of the Bessel function (30) and their asymptotic expressions for large order [53, there exists an integer $\ell_{0}$ such that

$$
j_{\ell+1 / 2,1}>v_{0}, \quad \ell \geq \ell_{0},
$$

and therefore $M=0$. Eventually, we shall reach a value $\ell_{\max } \geq \ell_{0}$ such that

(a) The inequality $(a)$ in (37) is just a consequence of $j_{\lambda, i}<j_{\lambda, i+1}$ given in (30).

(b) To prove the inequality (b) in (37) we first take into account that the bound states characterized by $n$ are determined, for a given $\ell$, by the function $\varphi_{\ell}(\varepsilon)$ restricted to the interval $\left(a_{n \ell}, b_{n \ell}\right)$, where

$$
a_{n \ell}=1-\frac{j_{\ell+1 / 2, n+1}^{2}}{v_{0}^{2}}, \quad b_{n \ell}=1-\frac{j_{\ell+1 / 2, n}^{2}}{v_{0}^{2}}, \quad n \in \mathbb{N}_{0} .
$$

We need to consider both functions $\varphi_{\ell}(\varepsilon), \varphi_{\ell+1}(\varepsilon)$, and therefore both intervals $\left(a_{n \ell}, b_{n \ell}\right)$, and $\left(a_{n(\ell+1)}, b_{n(\ell+1)}\right)$. Due to the properties of the zeros $j_{\ell+1 / 2, n}$ given in (30), the following relations are fulfilled:

$$
a_{n(\ell+1)}<a_{n \ell}, \quad b_{n(\ell+1)}<b_{n \ell} .
$$

Therefore, either (i) $b_{n(\ell+1)} \leq a_{n \ell}$, or (ii) $a_{n \ell}<b_{n(\ell+1)}$. - If (i) is true, $\left(a_{n(\ell+1)}, b_{n(\ell+1)}\right) \cap\left(a_{n \ell}, b_{n \ell}\right)=\emptyset$ so $-\varepsilon_{n \ell_{j}}<-\varepsilon_{n(\ell+1)_{j}}$ holds trivially.

- If (ii) is true, then we have three disjoint intervals: $\left(a_{n(\ell+1)}, a_{n \ell}\right),\left(a_{n \ell}, b_{n(\ell+1)}\right)$ and $\left(b_{n(\ell+1)}, b_{n \ell}\right)$. If $\varepsilon_{n \ell_{j}} \in\left(b_{n(\ell+1)}, b_{n \ell}\right)$ or $\varepsilon_{n(\ell+1)_{j}} \in\left(a_{n(\ell+1)}, a_{n \ell}\right)$, then it is obvious that $-\varepsilon_{n \ell_{j}}<-\varepsilon_{n(\ell+1)_{j}}$. However, if $\varepsilon_{n \ell_{j}}, \varepsilon_{n(\ell+1)_{j}} \in\left(a_{n \ell}, b_{n(\ell+1)}\right)$, the situation needs to be studied in detail. Let us prove first:

$$
\varphi_{\ell}\left(\varepsilon_{0}\right) \geq \varphi_{\ell+1}\left(\varepsilon_{0}\right), \quad \phi_{\ell}\left(\varepsilon_{0}\right)<\phi_{\ell+1}\left(\varepsilon_{0}\right),
$$

$\varepsilon_{0} \in\left(a_{n \ell}, b_{n(\ell+1)}\right)$. The first part results from B.10), since it holds for $v_{0} \in \mathbb{R}$, excluding the singularities

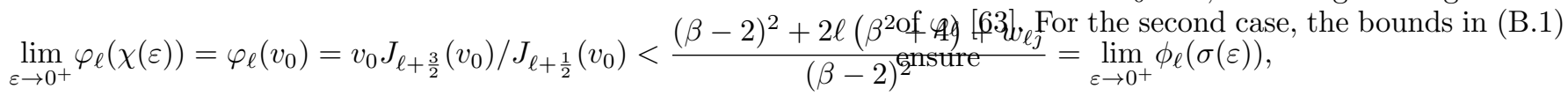
for all $\ell>\ell_{\max }$, hence $m=m^{\prime}=0$. In effect, the existence of $\ell_{\max }$ is a consequence of the dependence on the angular momentum of both sides in the previous inequality. It is clear that the right-hand side is a strictly increasing function with respect to $\ell$. In addition, using Theorem 3 of 63] it can be easily proved that the left-hand side fulfils

$$
\frac{\partial \varphi_{\ell}\left(v_{0}\right)}{\partial \ell} \leq 0
$$

In consequence, if none of the conditions $j_{\ell+1 / 2,1}<v_{0}$, $\varphi_{\ell}\left(v_{0}\right)>\phi_{\ell}\left(0^{+}\right)$hold, there is no bound state.

In order to complete the proof, we should consider a configuration in which an integer $s_{0} \in \mathbb{N}$ such that $v_{0}=j_{\ell+1 / 2, s_{0}}$ exists. In such a case the condition $\varphi_{\ell}\left(v_{0}\right)>$ $\phi_{\ell}\left(0^{+}\right)$is ill defined. Nevertheless, if $s_{0}>1$ we know the existence of at least one bound state. Thus, we have to consider the next value of the angular momentum for which $v_{0} \neq j_{\ell+1+1 / 2, s_{0}}$. If $s_{0}=1$ the bound state always exists, although, if $\phi_{\ell}\left(v_{0}\right)<0$, its energy can be below $-V_{0}$. Thus, we have to consider the next value of the angular momentum for which $v_{0} \neq j_{\ell+1+1 / 2,1}$.

Consequently, using 27], we reach

$$
\phi_{\ell+1}\left(\varepsilon_{0}\right)-\phi_{\ell}\left(\varepsilon_{0}\right)>1+\frac{w_{(\ell+1) j}-w_{\ell j}}{(2-\beta)^{2}} .
$$

In addition, for $\ell+1, j=(\ell+1)-1 / 2$ so, bearing in mind (8), the parameter $w_{(\ell+1) j}>0$. In a similar way, for $\ell, j=\ell+1 / 2$ and $w_{\ell}<0$. Consequently, the second inequality in $(\mathrm{B} .11$ is proved.

Now, we may prove $\varepsilon_{n(\ell+1)_{j}}<\varepsilon_{n \ell_{j}}$ by contradiction. Let us assume $\varepsilon_{n(\ell+1)_{j}} \geq \varepsilon_{n \ell_{j}}$. With B.11. and the monotonicity with respect to $\varepsilon$ above-mentioned, we find

$$
\phi_{\ell}\left(\varepsilon_{n \ell_{j}}\right) \leq \phi_{\ell}\left(\varepsilon_{n(\ell+1)_{j}}\right)<\phi_{\ell+1}\left(\varepsilon_{n(\ell+1)_{j}}\right)=\varphi_{\ell+1}\left(\varepsilon_{n(\ell+1)_{j}}\right) \leq
$$

From here it follows $\varphi_{\ell}\left(\varepsilon_{n \ell_{j}}\right) \neq \phi_{\ell}\left(\varepsilon_{n \ell_{j}}\right)$, which is clearly absurd because $\varepsilon_{n \ell_{j}}$ is a bound state, and the equality, 277, must be satisfied. 
(c) The inequality $(c)$ in $(37)$ is proved taking into account that the only dependence on $j$ in the secular equation (27) is through $w_{\ell j}$. As we have already pointed out, $w_{\ell(\ell-1 / 2)}>0>w_{\ell(\ell+1 / 2)}$. In consequence, $\phi_{\ell}$ is greater for $j=\ell-1 / 2$. Since $\varphi_{\ell}$ is independent of $j$ we only need to consider the interval $\left(a_{n \ell}, b_{n \ell}\right)$, for which the inequality is proved, as has been done before, by contradiction.

\section{References}

1. M. Belloni and R.W. Robinett, Phys. Rep. 50, 25122 (2014).

2. Yu N. Demkov and V.N. Ostrovskii, Zero-range Potentials and Their Applications in Atomic Physics (Plenum Press, New York, 1988).

3. S. Albeverio, F. Gesztesy, R. Hoegh-Krohn and H. Holden, Solvable Models in Quantum Mechanics, 2nd ed. (AMS Chelsea Publishing, Providence RI, 2004).

4. R. de L. Kronig and W.G. Penney, Proc. R. Soc. Lond. Ser. A 130, 499 (1931).

5. C. Kittel, Introduction to Solid State Physics, 8th ed. (John Wiley \& Sons, Inc., 2005).

6. F. Erman, M. Gadella, S. Tunalı, and H. Uncu, Eur. Phys. J. Plus 132, 352 (2017).

7. H. Uncu, D. Tarhan, E. Demiralp and Ö.E. Müstecaplıoğlu, Phys. Rev. A 76, 013618 (2007).

8. F. Ferrari, V.G. Rostiashvili and T.A. Vilgis, Phys. Rev. E 71, 061802 (2005).

9. I. Alvarado-Rodríguez, P. Halevi and J.J. SánchezMondragón, Phys. Rev. E 59, 3624 (1999); J.R. ZuritaSánchez and P. Halevi, Phys. Rev. E 61, 5802 (2000); Lin Ming-Chieh and Jao Ruei-Fu, Phys. Rev. E 74, 046613 (2006).

10. P. Kurasov, J. Math. Anal. Appl. 201, 297 (1996); S. Albeverio, L. Dabrowski and P. Kurasov, Lett. Math. Phys. 45, 33 (1998).

11. J.J. Alvarez, M. Gadella and L.M. Nieto, Int. J. Theor. Phys. 50, 2161 (2011); J.J. Alvarez, M. Gadella, L.P. Lara and F.H. Maldonado-Villamizar, Phys. Lett. A 377, 2510 (2013).

12. V.L. Kulinskii and D.Yu. Panchenko, Physica B: Cond. Matt. 472, 78 (2015); Ann. Phys. 404, 47 (2019).

13. S. Albeverio and P. Kurasov, Singular Perturbations of Differential Operators (Lecture Note Series) 271 (Cambridge University Press, Cambridge, 1999).

14. M. Gadella M, J. Negro J and L.M. Nieto, Phys. Lett. A 373, 1310 (2009).

15. S. Albeverio, S. Fassari and F. Rinaldi, J. Phys. A: Math. Theor. 49, 025302 (2016).

16. S. Fassari, M. Gadella, M.L. Glasser and L.M. Nieto, Ann. Phys. 389, 48 (2018).

17. M. Gadella, M.L. Glasser and L.M. Nieto, Int. J. Theor. Phys. 50, 2144 (2011); Int. J. Theor. Phys. 50, 2191 (2011).

18. J.M. Muñoz-Castañeda and J.M. Mateos-Guilarte, Phys. Rev. D 91, 025028 (2015).

19. N. Graham et al., Nucl. Phys. B 677379 (2004).

20. G. Barton, J. Phys. A: Math. Gen. 37, 1011 (2004).

21. A.V. Zolotaryuk, Phys. Rev. A 87, 052121 (2013).

22. A.V. Zolotaryuk and Y. Zolotaryuk, J. Phys. A: Math. Theor. 48, 035302 (2015).

23. M. Calçada, J.T. Lunardi, L.A. Manzoni and W. Monteiro, Front. Phys. 2, 23 (2014).
24. J.I. Díaz, J. Negro, L.M. Nieto and O. Rosas-Ortiz, J. Phys. A: Math. Gen. 32, 8447 (1999); J. Negro, L.M. Nieto and O. Rosas-Ortiz, Foundations of Quantum Physics, R. Blanco et al. ed., p 259 (CIEMAT/RSEF, Madrid, 2002). 25. D.J. Fernández C., M. Gadella and L.M. Nieto, SIGMA 7, 029 (2011).

26. E. Díaz-Bautista and D.J. Fernández C., Eur. Phys. J. Plus 132, 499 (2017).

27. J. Mateos Guilarte, J.M. Muñoz Castañeda, and A. Moreno Mosquera, Eur. Phys. J. Plus 130, 48 (2015).

28. J. Mateos Guilarte and A. Moreno Mosquera, Eur. Phys. J. Plus 132, 93 (2017).

29. R. de la Madrid, Nucl. Phys. A 962, 24 (2017).

30. R.M. Id Betan and R. de la Madrid, Nucl. Phys. A 970, 398 (2018).

31. F.I. Sharrad, A.A. Okhunov, H.Y. Abdullah, and H.A. Kassim, Int. J. Phys. Sci., 7(38), 5449 (2012).

32. T. Vertse, K.F. Pal and Z. Balogh, Comp. Phys. Comm. 27, 309 (1982).

33. B. Gyarmati, K.F. Pal and T. Vertse, Phys. Lett. 104, 177 (1981).

34. R. Machleidt, Computational Nuclear Physics Nuclear Reactions, K. Langanke, J.A. Maruhn and S.E. Koonin eds.(Springer, New York, 1993) p. 1.

35. R.D. Woods and D.S. Saxon, Phys. Rev. 95, 577 (1954).

36. A. Bohr and B.R. Mottelson, Nuclear Structure, vol 1 (Benjamin, New York, 1969).

37. A. Van Der Woude and B.J. Verhaar (eds.), International Conference on Nuclear Structure: Proceedings of the International Conference on Nuclear Structure (9th EPS Nuclear Physics Divisional Conference) (Elsevier Science, Saint Louis, 2016).

38. N. Schwierz, I. Wiedenhöver and A. Volya, Parameterization of the Woods-Saxon Potential for Shell-Model Calculations, arXiv:0709.3525 [nucl-th] (2007).

39. J. Suhonen, From Nucleons to Nucleus (Springer, New York, 2007).

40. M.G. Mayer, Phys. Rev. 75, 1969 (1949).

41. O. Haxel, J.H.D. Jensen and H.E. Suess, Phys. Rev. 75, 1766 (1949).

42. L. Schwartz, Théorie des Distributions (Hermann, Paris, 1966).

43. W.O. Amrein, J.M. Jauch and K.B. Sinha, Scattering Theory in Quantum Mechanics (Benjamin, Reading Massachusetts, 1977)

44. J.M. Muñoz-Castañeda, L.M. Nieto and C. Romaniega, Ann. Phys. 400, 246 (2019).

45. M. Gadella, J.M. Mateos-Guilarte, J.M. Muñoz-Castañeda and L.M. Nieto, J. Phys. A: Math. Theor. 49, 015204 (2016).

46. V. Bargmann, Proc. Nat. Acad. Sci. 38, 961 (1952).

47. J. Schwinger, Proc. Nat. Acad. Sci. 47, 122 (1961).

48. A. Galindo and P. Pascual, Quantum Mechanics I (Texts and Monographs in Physics) (Springer-Verlag, Berlin, 1990).

49. J.W. Mayer and J.H.D. Jensen, Elementary theory of nuclear shell structure (Wiley, New York, 1955).

50. G.F. Bertsch, The practioner's shell model (North Holland, New York, 1972).

51. J. Mur-Petit, A. Polls and F. Mazzanti, Am. J. Phys. 70, 808 (2002).

52. A. Galindo and R. Tarrach, Ann. Phys. 173, 430 (1987).

53. F.W.J. Olver, NIST Handbook of Mathematical Functions (Cambridge Univ. Press, New York, 2010). 
54. See the following official web page: https://www.nndc.bnl.gov/nudat2/chartNuc.jsp

55. D.A. Bromley, Comments Nucl. Part. Phys. 2, 151 (1968).

56. M. Reed and B. Simon, Analysis of Operators (Academic Press, New York, 1978).

57. H.M. Nussenzveig, Causality and Dispersion Relations (Academic, New York, 1972).

58. A. Bohm, Quantum Mechanics: Foundations and Applications, 3rd ed (Springer, Berlin, 1993).

59. M. Reed and B. Simon, Fourier Analysis. Self Adjointness (Academic Press, New York, 1975).

60. M. Abramowitz and I.A. Stegun, Handbook of Mathematical Functions (Dover, New York, 1972).

61. J. Segura, J. Math. Appl. 374, 516 (2011).

62. A. Laforgia and P. Natalini, J. Ineq. Appl. 2010253035 (2010).

63. L.J. Landau, J. Math. Anal. Appl. 240, 174 (1999).

64. G. Levai, A. Baran, P. Salamon, T. Vertse, Phys. Lett. A, 381, 1936-1942 (2017).
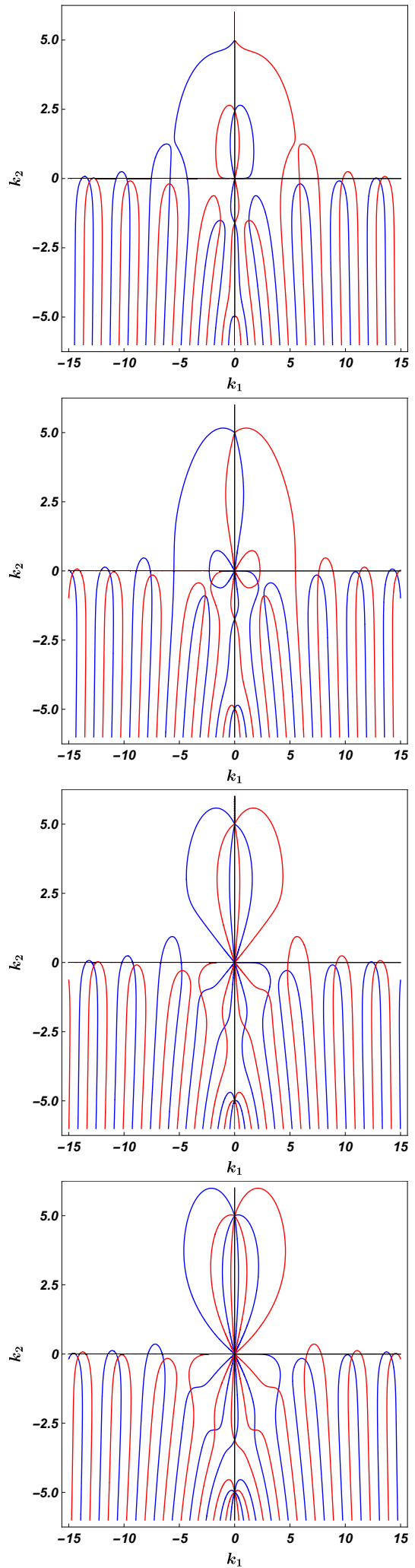

Fig. 3. Annihilation of 45: in blue the real part, in red the imaginary part. Bound states and resonances correspond to the intersection of red and blue curves. From top to bottom and left to right the curves for $\ell=1,2,3,4$. The relevant parameters are chosen to be $v_{0}=5, w_{\ell j}=10$ and $\beta=1$. 
16 Romaniega, Gadella, Id Betan \& Nieto: An approximation to Woods-Saxon potential based on a contact interaction

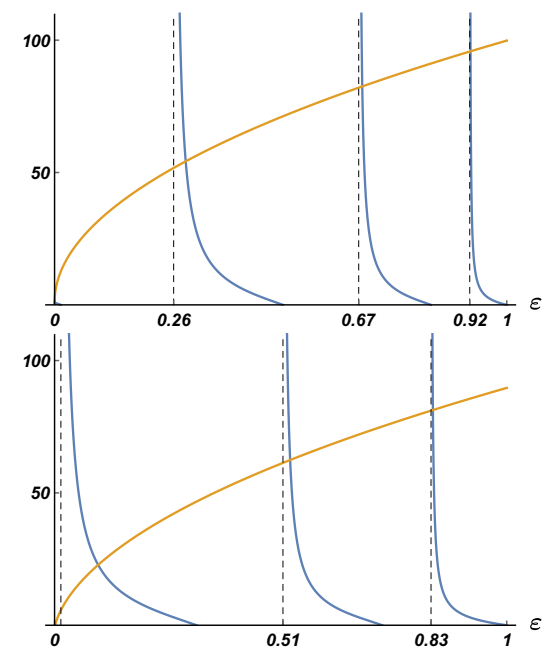

Fig. 4. Results for the isotope ${ }^{209} \mathrm{~Pb}$. In blue the left-hand side $\varphi_{\ell}$ of 27 in yellow the right-hand side $\phi_{\ell}$ for $\beta=1$. On the left the case $\ell=0$, on the right the case $\ell=1$ and $j=3 / 2$. 